\title{
Gait speed, cognition and falls in people living with mild-to-moderate Alzheimer disease: data from NILVAD
}

\author{
Adam H. Dyer ${ }^{1,2^{*}}$, Brian Lawlor ${ }^{2,3}$, Sean P. Kennelly ${ }^{1,2}$ and for the NILVAD Study Group
}

\begin{abstract}
Background: Previous evidence suggests that slower gait speed is longitudinally associated with cognitive impairment, dementia and falls in older adults. Despite this, the longitudinal relationship between gait speed, cognition and falls in those with a diagnosis of dementia remains poorly explored. We sought to assess this longitudinal relationship in a cohort of older adults with mild to-moderate Alzheimer Disease (AD).
\end{abstract}

Methods: Analysis of data from NILVAD, an 18-month randomised-controlled trial of Nilvadipine in mild to moderate AD. We examined: (i) the cross-sectional (baseline) association between slow gait speed and cognitive function, (ii) the relationship between baseline slow gait speed and cognitive function at 18 months (Alzheimer Disease Assessment Scale, Cognitive Subsection: ADAS-Cog), (iii) the relationship between baseline cognitive function and incident slow gait speed at 18 months and finally (iv) the relationship of baseline slow gait speed and incident falls over the study period.

Results: Overall, one-tenth $(10.03 \%, N=37 / 369)$ of participants with mild-to-moderate AD met criteria for slow gait speed at baseline and a further 14.09\% ( $N=52 / 369)$ developed incident slow gait speed at 18 months. At baseline, there was a significant association between poorer cognition and slow gait speed (OR 1.05, 95\% Cl 1.01-1.09, $p=0.025$ ). Whilst there was no association between baseline slow gait speed and change in ADAS-Cog score at 18 months, a greater cognitive severity at baseline predicted incident slow gait speed over 18 months (OR 1.04, 1.01-1.08, $p=0.011$ ). Further, slow gait speed at baseline was associated with a significant risk of incident falls over the study period, which persisted after covariate adjustment (IRR 3.48, 2.05-5.92, p<0.001).

Conclusions: Poorer baseline cognition was associated with both baseline and incident slow gait speed. Slow gait speed was associated with a significantly increased risk of falls over the study period. Our study adds further evidence to the complex relationship between gait and cognition in this vulnerable group and highlights increased falls risk in older adults with $\mathrm{AD}$ and slow gait speed.

Trial registration: Secondary analysis of the NILVAD trial (Clincaltrials.gov NCT02017340; EudraCT number 2012-002764-27). First registered: 20/12/2013.

Keywords: Dementia, Alzheimer disease, Cognition, Falls

\footnotetext{
* Correspondence: dyera@tcd.ie

'Department of Age-Related Healthcare, Tallaght University Hospital, Dublin, Ireland

${ }^{2}$ Department of Medical Gerontology, School of Medicine, Trinity College

Dublin, Dublin 2, Ireland

Full list of author information is available at the end of the article
}

(c) The Author(s). 2020 Open Access This article is licensed under a Creative Commons Attribution 4.0 International License, which permits use, sharing, adaptation, distribution and reproduction in any medium or format, as long as you give appropriate credit to the original author(s) and the source, provide a link to the Creative Commons licence, and indicate if changes were made. The images or other third party material in this article are included in the article's Creative Commons licence, unless indicated otherwise in a credit line to the material. If material is not included in the article's Creative Commons licence and your intended use is not permitted by statutory regulation or exceeds the permitted use, you will need to obtain permission directly from the copyright holder. To view a copy of this licence, visit http://creativecommons.org/licenses/by/4.0/ The Creative Commons Public Domain Dedication waiver (http://creativecommons.org/publicdomain/zero/1.0/) applies to the data made available in this article, unless otherwise stated in a credit line to the data. 


\section{Background}

Gait disorders affect a significant number of communitydwelling older adults and have recently been highlighted as a potential indicator of later cognitive decline. Gait disorders are believed to affect up to one third of older adults aged $>70$ years and may be divided based on the particular sensorimotor deficit involved: (i) lower level gait disorders such as osteoarthritis, (ii) mid-level gait disorders which result from a focal neurological lesion such as that seen in Parkinson's Disease and (iii) higher level gait disorders with no demonstrable deficits in the pyramidal/extrapyramidal/sensory or cerebellar systems (and may be particularly related to cerebrovascular disease) [1-3].

Not only does slow gait speed predict a decline in functional status, but has also been linked with adverse outcomes including mortality in older adults [4-9]. Further, there is now a significant body of evidence linking slow gait speed, and in particular a decline in gait speed, with later risk of cognitive decline and dementia. For instance, in a recently published study from the English Longitudinal Study of Aging, both baseline gait speed and change in gait speed was associated with dementia risk over a follow up of $>10$ years [10]. This follows a large body of evidence indicating the strong relationship between slow gait speed and later cognitive decline [11-16].

Whilst the evidence for the relationship between slow gait speed and later dementia risk is mounting, the relationship between gait speed and cognitive trajectories in those with Alzheimer Disease (AD) is less clear. Whilst a handful of studies have evaluated the association between gait speed and dementia in cross-section [17-19], longitudinal analyses are limited. A notable study has demonstrated a decline in gait speed associated with clinical progression in those with Mild Cognitive Impairment (MCI) [20]. In the only longitudinal study of gait speed in those with a diagnosis of dementia, baseline gait-speed was associated with a decline in verbal fluency over a 1 year period, but not in overall cognition [21]. Thus, whilst the cross-sectional relationship between gait and cognition in dementia has been demonstrated, the longitudinal relationship between slow gait speed and cognitive function in AD remains unclear.

There is a significant body of evidence reporting that older adults with a diagnosis of $\mathrm{AD}$ are at greater risk of falls than their age matched counterparts without dementia [22-26]. The increased risk represents the interaction of several risk factors such as autonomic symptoms, physical activity profile, orthostatic hypotension and polypharmacy [24]. Several studies have reported associations between spatiotemporal gait parameters and falls risk in older adults with dementia [27], however there are also conflicting results. Many studies have taken place in nursing home or long term care facilities and the association has been less-well explored in community dwelling older adults with a diagnosis of $\mathrm{AD}$, particularly those with mild-to-moderate AD [27]. Further, most studies on community dwelling older adults with $\mathrm{AD}$ have either occurred in cross-section or retrospectively with $<12$ months follow up [27].

Based on the above evidence and the known adverse effects experienced by those with slow gait speed, it follows that slow gait speed may be an indicator of which patients with $\mathrm{AD}$ are particularly vulnerable of further cognitive decline and adverse outcomes such as falls. In the current study, we aimed to assess the relationships between (i) baseline cognition and baseline slow gait speed in mild-moderate AD, (ii) baseline slow gait speed and cognitive decline at 18 months (iii) baseline cognitive impairment and incident slow gait speed at 18 months (decline in gait speed) and (iv) the longitudinal relationship between baseline slow gait speed and incident falls over 18 months in older adults with a diagnosis of mild-to moderate AD.

\section{Methods \\ Setting}

The current study was embedded within NILVAD, a randomised controlled trial of the antihypertensive Nilvadipine in mild-to-moderate Alzheimer Disease (AD). The trial's primary outcome of a therapeutic effect of Nilvadipine in mild/ moderate AD was not achieved. Briefly, participants were recruited from 23 centres in 9 European countries (Clincaltrials.gov NCT02017340; EudraCT number 2012-002764-27). The full trial protocol, the sub-study protocol in addition to the main trial results have been published elsewhere [28-30]. Ethical approval for the trial was granted from the appropriate National Competent Authorities, Independent Ethics Committees and Institutional Review Boards for all study sites.

Participants once enrolled were randomized to either Nilvadapine $8 \mathrm{mg}$ or placebo for the 18 month duration of the study.

\section{Inclusion/exclusion criteria}

The inclusion/exclusion criteria for the NILVAD trial have been previously published [28]. Of note, included participants were those aged $>50$ years who met the National Institute of Neurological and Communicative Disorders and Stroke/Alzheimer's Disease Criteria: NINCDS-ADRDA for AD. Criteria for inclusion was a diagnosis of mild-to-moderate $\mathrm{AD}$, defined as a Mini-Mental State (MMSE) Score between 12 and 26 at trial enrolment and a diagnosis of $\mathrm{AD}$ as per the NINCDS-ADRDA criteria. Further, those included had a Mini-Mental State (MMSE) Score between 12 and 26 at trial enrolment, meeting criteria for mild to moderate AD.

Relevant exclusion criteria to the current analysis include a comorbid neurological condition such as Parkinson's Disease 
or Huntington's Disease. Similarly, participants with a history of significant head trauma, known structural brain abnormalities or any other condition known to interfere with cognitive function were excluded. Patients meeting criteria for a substance use disorder were also excluded as were participants with significant cardiovascular disease. For full exclusion criteria, readers are directed to the initial trial protocol.

\section{Gait measurement}

Gait speed was measured as part of the NILVAD frailty sub-study, the protocol of which has been previously published [28]. Briefly, gait speed was measured both at baseline and 18 months. Four markings were placed on an even floor with adhesive tape over a distance of $6 \mathrm{~m}$ total. The markings were spaced as follows (i) first mark at start, (ii) second mark at $1 \mathrm{~m}$, (iii) third mark $4 \mathrm{~m}$ from mark 2 and (iv) fourth mark $1 \mathrm{~m}$ from mark 3. Participants were asked to stand at mark 1 and then instructed to walk from mark 1 to mark 4 at normal walking speed. The stopwatch was started once the participant's first foot touched/ crossed mark two and was stopped once the participant's last foot crossed mark 3. A stopwatch accurate to two decimal places was used. Thus the total speed represented the time for the participant to walk $4 \mathrm{~m}$. If the total time was $>6 \mathrm{~s}$ to complete this $4 \mathrm{~m}$ walk $\left(<0.67 \mathrm{~ms}^{-1}\right)$, gait speed was recorded as slow gait speed. This pre-specified binary cut-off was applied as per previous literature and included in the initial sub-study protocol $[23,28]$.

Baseline slow gait speed was considered as those below this cut-off at trial initiation and incident slow gait speed defined as those below this cut off at 18 months who did not meet the slow great criteria at baseline.

\section{Cognitive function and dementia severity assessment}

The Alzheimer's Disease Assessment Scale, Cognitive Subsection (ADAS-Cog) was used to assess cognition and was the primary cognitive endpoint in the NILVAD trial. For the current study, we considered ADAS-Cog at initial visit to be baseline ADAS-Cog and analysed the change in ADAS-Cog at 18 months as the main cognitive outcome. ADAS-Cog sub-scores analysed included the following: word recall task, naming, commands, constructional praxis, delated word recall, ideational praxis, orientation, word recognition task, spoken language ability, comprehension, word finding difficult in spontaneous speech, remembering test instructions.

Dementia severity at baseline and at 18-months was assessed using the Clinical Dementia Rating scale sum of boxes (CDR-Sb) score.

\section{Medical history and regular medications}

A comprehensive medical history was taken from participants/carers at time of study enrolment and updated on follow up visits. A list of concomitant medications was also obtained at time of study enrolment and reviewed at follow up visits in order to identify any changes in concomitant medication usage. Medications were classified according to Anatomic Therapeutic Classification (ATC) codes to ensure consistency. For the current analysis, only medications taken for the entire 18-month study duration were considered. Short term and historical medication use were excluded.

\section{Statistical analysis}

STATA V15.0 (Stata Corp, College Station, Texas, USA) was used for all analysis in the current study, with the significance level considered as $p<0.05$. Baseline descriptive statistics are reported as means with standard deviations where parametric and medians with Interquartile Ranges (IQR) where appropriate. Univariate analysis of between group differences employed T-tests, Wilcoxon rank-sum tests and Chi-square tests. Logistic regression was used to assess predictors of slow gait speed at baseline and adjusted Odds Ratios (OR), 95\% Confidence Intervals (CI) and $p$-values presented as appropriate. Change in ADAS-Cog Scores was considered the difference between the baseline ADAS-Cog score and ADAS-Cog score at 18 months, with an increasing score indicating greater cognitive decline.

In the current analysis, we sought to assess the longitudinal relationship between slow gait speed and cognitive function in older adults with mild-to-moderate Alzheimer Disease. Our research questions were as follows: (i) is there an association at baseline between slow gait speed and baseline cognitive function?, (ii) does slow gait speed at baseline predict greater cognitive decline at 18 months in mild-to-moderate AD?, (iii) does cognitive function at baseline predict the development of slow gait speed at 18 months (incident slow gait speed)?, and finally, (iv) does slow gait speed predict falls in those with $\mathrm{AD}$ over an 18-month period.

To assess the cross-sectional relationship at baseline between cognitive function (independent variable, linear) and slow gait speed (dependent variable; binary), we used multivariate logistic regression adjusting for important confounders known to influence gait speed and/ or cognition. We adjusted for age, gender, Body Mass Index, study group (Nilvadipine vs.placebo), diagnosis duration, symptom duration, years of formal education, total number of medications, antidepressant and benzodiazepine use, total medical comorbidities, diabetes, hypertension, arthritis, baseline ADAS-Cog and baseline CDR-Sb.

In order to assess whether baseline slow gait speed (independent variable; categorical) was associated with cognitive outcomes at 18 months (dependent variable; linear), mixed effects linear regression was used with country as a random effect. The association was tested unadjusted in the first 
instance (model 1). The association was then tested controlling for important demographic and AD-related covariates including baseline cognitive function (ADAS-Cog Score), age, gender, study arm, body mass index, years of formal education and diagnosis duration (model 2). Finally we incorporated total number of medical comorbidities and total number of medications (model 3).

For assessment of whether baseline cognition (independent variable; linear) was associated with incident slow gait speed (dependent variable; categorical), logistic regression was used Again the association was tested unadjusted followed by adjustment for baseline ADAS-Cog, age, gender, study arm, body mass index, years of formal education, diagnosis duration (model 2) followed by robust adjustment (model 3) for total number of medical conditions, diabetes mellitus, arthritis, total number of medications, antidepressant use and benzodiazepine use based on known impact on gait speed.

Finally, in order to assess the relationship between slow gait speed (independent variable; categorical) and falls (dependent variable; count), a Poisson regression was used, again unadjusted in the first instance, followed by adjustment for baseline ADAS-Cog, age, gender, study arm, body mass index, years of formal education, diagnosis duration (model 2) in addition to total medical comorbidities and total number of medications (model 3).

Analyses were then repeated by ADAS-Cog sub-scores for each task, with the number of errors on each task analysed by Poisson regression using the same covariates the above models. Finally, we repeated the above models using the CDR-Sb as the dependent variable in order to assess the relationship between slow gait speed and dementia severity at baseline at 18 months.

In all above analyses, the following data were categorical: gender, group (Nilvadipine vs. placebo), slow gait speed (present vs absent), antidepressant use (user vs non-user), benzodiazepine use (user vs non-user), diabetes (present vs absent), arthritis (present vs absent), hypertension (present vs absent). All other variables were continuous as detailed above.

\section{Results}

\section{Participant characteristics}

Of those randomized to either Nilvadipine or placebo at baseline $(N=511), 465$ participants had a gait speed measurement performed. Of these, 369 had full clinical and 18month follow up data obtained including repeat gait speed and cognitive assessment at 18 months. This population constituted those included in the current analysis. Mean age of included participants was $72.8 \pm 8.06$ and $63.14 \%$ $(N=233 / 369)$ were female. Overall, $50.68 \%(N=187 / 369)$ were assigned to the treatment group. Median duration of AD symptoms was 3.65 years (IQR: 2.26-5.18) and duration since diagnosis was 0.91 years (IQR: $0.42-2.12$ ). At baseline, mean ADAS-Cog was $32.97 \pm 9.81$ and mean $\mathrm{CDR}-\mathrm{Sb}$ was $4.82 \pm 2.52$.

\section{Baseline association between slow gait speed and cognition}

At baseline, one-tenth $(10.03 \%, N=37 / 369)$ of participants had slow gait speed defined as described above. On univariate analysis, those with slow gait speed were older, had a higher BMI, fewer years of education, greater number of total medications and comorbidities, poorer baseline cognition rated using the ADAS-Cog and greater dementia severity rated using the CDR-Sb (all $p<0.05$, see Table 1 ). On multivariate analysis controlling for all covariates, increasing age (OR 1.10, 1.04-1.17, $p<0.001)$ and poorer baseline cognition on the ADAS-Cog (OR 1.05, 1.01-1.09, $p=0.025)$ were associated with greater risk of baseline slow gait speed (See Table 2). Similarly, there was a nonsignificant statistical trend for greater dementia severity using the CDR-Sb as a predictor of slow gait speed under this model (OR 1.19, 0.98-1.45, $p=0.08$ ). On analysis of ADAS-Cog subset-scores, there were no specific associations between gait speed and separate cognitive domain subset-scores at 18 months.

\section{Change in ADAS-cog scores over 18 months}

Overall, the mean ADAS-Cog in the included cohort increased by 8.87 ( \pm 9.18$)$, indicating both a clinically significant cognitive decline [31] and a statistically significant progression $(t=-18.56, p<0.001)$.

\section{Baseline slow gait speed and cognitive decline at 18 months}

Unadjusted, there was no association between baseline slow gait speed and ADAS-Cog scores at 18 months $(\beta$ : $1.49,-4.61-1.63, p=0.35)$. There was no association on controlling for baseline ADAS-Cog, age, gender, study arm, BMI, education and diagnosis duration $(\beta:-0.63$, $3.69-2.42, p=0.69)$. Further, following further adjustment for important medical comorbidities and concomitant medication use, there was no association between baseline gait speed and ADAS-Cog scores at 18 months $(\beta:-0.80,-3.86-2.25, \mathrm{p}=0.69)$ (Table 3). Under the same models, there was no association between slow gait speed at baseline and CDR-Sb score at 18 months either unadjusted $(\beta: 0.23,-0.79-1.24, p=0.66)$, or under model $2(\beta$ : $0.11,-0.92-1.14, p=0.84)$ or model $3(\beta$ : $0.16,-0.92-1.15, p=0.83)$.

\section{Baseline cognition and incident slow gait speed at 18 months}

Overall, $14.09 \%(N=52 / 369)$ of participants with mild to moderate $\mathrm{AD}$ met the criteria for incident slow gait speed at 18 month follow up (having had normal gait speed at baseline). Overall, those participants meeting 
Table 1 Baseline Differences between Those with Mild to Moderate AD with Slow Gait Speed at Baseline and Those Without (Univariate Analysis). ADAS-Cog = Alzheimer Disease Assessment Scale - Cognitive Subsection; CDR-Sb: Clinical Dementia Rating, Sum of Boxes, BMl: Body Mass Index

\begin{tabular}{|c|c|c|c|}
\hline Characteristic & $\begin{array}{l}\text { No Slow Gait Speed } \\
(N=332)\end{array}$ & $\begin{array}{l}\text { Slow Gait Speed } \\
(N=37)\end{array}$ & $P$-Value \\
\hline Age, mean (SD) & $72.25(8.02)$ & $77.68(6.75)$ & $<0.001^{*}$ \\
\hline Gender, female (\%) & $207(62.35 \%)$ & $26(70.27 \%)$ & 0.343 \\
\hline BMI, mean (SD) & $25.44(4.23)$ & $27.97(5.56)$ & $0.005^{*}$ \\
\hline Group, nilvadipine (\%) & $169(50.9 \%)$ & $18(48.65 \%)$ & 0.795 \\
\hline Diagnosis Duration, median (IQR) & $0.90(0.43-2.22)$ & $1.01(0.48-1.71)$ & 0.687 \\
\hline Symptom Duration, median (IQR) & $3.69(2.20-5.25)$ & $3.45(2.50-4.28)$ & 0.689 \\
\hline Education, median (IQR) & $16(14-18)$ & $14(12-16)$ & $0.003^{*}$ \\
\hline Total Medications, median (IQR) & $5(3-6.5)$ & $5(4-8)$ & $0.022^{*}$ \\
\hline Antidepressant Use, N (\%) & $126(37.95 \%)$ & $18(48.65 \%)$ & 0.206 \\
\hline Benzodiazepine Use, N (\%) & $40(12.05 \%)$ & $3(8.11 \%)$ & 0.479 \\
\hline Total Comorbidities, median (IQR) & $4(2-5)$ & $4(3-6)$ & $0.009 *$ \\
\hline Diabetes, N (\%) & $22(6.63 \%)$ & $5(13.51 \%)$ & 0.297 \\
\hline Hypertension, N (\%) & $94(28.40 \%)$ & $14(37.84 \%)$ & 0.232 \\
\hline Arthritis, N (\%) & $26(7.83 \%)$ & $4(10.81)$ & 0.529 \\
\hline Baseline ADAS-Cog, mean (SD) & $32.49(9.49)$ & $37.29(11.56)$ & $0.002^{*}$ \\
\hline Baseline CDR-Sb, mean (SD) & $4.67(2.44)$ & $6.13(2.84)$ & $0.004^{*}$ \\
\hline
\end{tabular}

Table 2 Multivariate Analysis of Slow Gait Speed at Baseline. At baseline, increasing age and poorer scores on the ADAS-Cog at baseline was associated with significantly greater likelihood of slow gait speed (dependent variable) at baseline. ADAS-Cog = Alzheimer Disease Assessment Scale - Cognitive Subsection; CDR-Sb: Clinical Dementia Rating, Sum of Boxes, BMI: Body Mass Index

\begin{tabular}{lll}
\hline Characteristic & OR $(95 \%$ Cl $)$ & $P$-Value \\
\hline Age & $1.10(1.04-1.17)$ & $<\mathbf{0 . 0 0 1 *}$ \\
Gender & $1.14(0.47-2.75)$ & 0.773 \\
BMI & $1.08(0.99-1.16)$ & 0.078 \\
Group & $1.00(0.45-2.21)$ & 0.996 \\
Diagnosis Duration & $0.88(0.64-1.20)$ & 0.417 \\
Symptom Duration & $0.94(0.78-1.14)$ & 0.541 \\
Education (Years) & $0.93(0.83-1.04)$ & 0.214 \\
Total Medications & $1.07(0.91-1.25)$ & 0.421 \\
Antidepressant Use & $1.53(0.69-3.41)$ & 0.298 \\
Benzodiazepine Use & $0.36(0.07-1.60)$ & 0.178 \\
Total Comorbidities & $1.10(0.93-1.31)$ & 0.270 \\
Diabetes & $0.96(0.30-3.11)$ & 0.951 \\
Hypertension & $1.76(0.77-3.98)$ & 0.179 \\
Arthritis & $0.86(0.23-3.20)$ & 0.820 \\
Baseline ADAS-Cog & $1.05(1.01-1.09)$ & $\mathbf{0 . 0 2 5 *}$ \\
Baseline CDR-Sb & $1.19(0.98-1.45)$ & 0.081 \\
\hline
\end{tabular}

criteria for incident slow gait speed had higher baseline ADAS-Cog scores, however this was not a statistically significant or clinically meaningful difference $(36.47+/-9.44$ vs $36.08+/-11.42, \mathrm{t}=-0.23, p=0.82$ ) [31]. Unadjusted, ADAS-Cog score at baseline was associated with a greater likelihood of incident slow gait speed at 18 months (OR: $1.04,1.01-1.07, p=0.015)$. This association persisted after controlling for age, gender, BMI, study group, years of education and diagnosis duration (OR: 1.04, 1.01-1.08, $p=0.009$ ). Further, after robust covariate adjustment for total medical comorbidities, diabetes, arthritis, total number of medications, benzodiazepines and antidepressant use, the association remained significant (OR: 1.04, 1.011.08, $p=0.011$ ) (see Table 4). Further, dementia severity at baseline rated using the CDR-Sb was significant under the same models with associations seen unadjusted (OR: 1.17, $1.05-1.30, p=0.003$ ) in addition to under models 2 (OR: $1.16,1.03-1.30, p=0.018$ ) and 3 (OR: $1.15,1.01-1.30, p=$ $0.031)$.

Cognitive Scores (ADAS-Cog) of those at baseline with baseline slow gait speed and normal gait speed in addition to those meeting criteria for incident slow gait speed are detailed below in Table 5 .

\section{Baseline slow gait speed and incident falls over an 18 month duration}

Overall, 57 (15.45\%) participants experienced at least one fall over the 18 month duration of the trial. A small 
Table 3 Analysis of The Relationship Between Baseline Slow Gait Speed and Cognition at 18 Months (ADAS-Cog) in Mild to Moderate AD. Under unadjusted or adjusted models, there was no association between baseline slow gait speed and cognition at 18 months (dependent variable). ADAS-Cog = Alzheimer Disease Assessment Scale - Cognitive Subsection

\begin{tabular}{|c|c|c|c|c|c|c|}
\hline Predictor & Model 1 & & Model 2 & & Model 3 & \\
\hline & $\beta$-Coef $(95 \% \mathrm{Cl})$ & $P$ & $\beta$-Coef $(95 \% \mathrm{Cl})$ & $P$ & $\beta$ Coef $(95 \% \mathrm{Cl})$ & $P$ \\
\hline Baseline Slow Gait Speed & $-1.49(-4.61,1.63)$ & 0.35 & $-0.63(-3.69,2.42)$ & 0.69 & $-0.80(-3.86,2.25)$ & 0.606 \\
\hline Baseline ADAS-Cog & & & $0.22(0.13,0.31)$ & $<0.001^{*}$ & $0.22(0.13,0.32)$ & $<0.001^{*}$ \\
\hline Age & & & $-0.21(-0.32,-0.10)$ & $<0.001 *$ & $-0.22(-0.34,-0.10)$ & $<0.001 *$ \\
\hline Gender (Female) & & & $0.94(-2.80,0.92)$ & 0.321 & $0.97(-2.83,0.90)$ & 0.310 \\
\hline Study Arm & & & $-0.73(-2.49,1.02)$ & 0.414 & $-0.70(-2.46,1.06)$ & 0.437 \\
\hline Body Mass Index & & & $-0.25(-0.46--0.04)$ & $0.018^{*}$ & $-0.27(-0.48--0.06)$ & $0.011^{*}$ \\
\hline Education (Years) & & & $0.13(-0.11-0.37)$ & 0.291 & $0.14(-0.10-0.38)$ & 0.244 \\
\hline Diagnosis Duration & & & $-0.67(-1.22--0.12)$ & 0.017 & $-0.68(-1.22--0.12)$ & 0.015 \\
\hline Total Medications & & & & & $0.17(-0.18-0.52)$ & 0.345 \\
\hline Total Comorbidities & & & & & $0.08(-0.33-0.49)$ & 0.697 \\
\hline
\end{tabular}

minority, 21 (5.69\%), experienced recurrent (>1) falls over the study period. Unadjusted, slow baseline gait speed was associated with increasing likelihood of falls (IRR: $3.18,1.97-5.11, p<0.001)$. Controlling for demographic and baseline $\mathrm{AD}$ variables, this association persisted (IRR: 3.27, 1.96-5.50, $p<0.001$ ). Finally, after robust adjustment for important comorbidities and medications, the association remained significant (IRR: 3.48, $2.05-5.92, p<0.001)$. See Table 6 for details. Under all models, slow gait speed was the strongest independent predictor of falls over the 18 month duration.

\section{Discussion}

In this study of older adults mild-to-moderate AD, greater cognitive impairment at baseline was significantly associated with both baseline slow gait speed and incident slow gait speed at 18 months. There was no association between baseline slow gait speed and longitudinal cognitive performance at 18 months. This is despite a clinically meaningful cognitive decline experienced by the cohort overall (with the mean ADAS-Cog score increasing by a mean of 8.87 points) [32]. Further, slow gait speed at baseline was associated with a more

Table 4 Association between Baseline Cognition (ADAS-Cog) and incident Slow Gait Speed at 18 Months in Mild to Moderate AD. Under all models, baseline cognition was significantly associated with incident slow gait speed (dependent). ADAS-Cog = Alzheimer Disease Assessment Scale - Cognitive Subsection

\begin{tabular}{|c|c|c|c|c|c|c|}
\hline Predictor & Model 1 & & Model 2 & & Model 3 & \\
\hline & OR $(95 \% \mathrm{Cl})$ & $P$ & OR $(95 \% \mathrm{Cl})$ & $P$ & OR $(95 \% \mathrm{Cl})$ & $P$ \\
\hline Baseline ADAS-Cog & $1.04(1.01,1.07)$ & $0.015^{*}$ & $1.04(1.01-1.08)$ & $0.009^{*}$ & $1.04(1.01-1.08)$ & $0.011^{*}$ \\
\hline Age & & & $1.10(1.05-1.15)$ & $<0.001^{*}$ & $1.10(1.05-1.16)$ & $<0.001 *$ \\
\hline Gender (Female) & & & $0.61(0.32-1.18)$ & 0.143 & $0.63(0.32-1.24)$ & 0.178 \\
\hline Study Arm & & & $0.82(0.44-1.54)$ & 0.534 & $0.70(0.37-1.36)$ & 0.294 \\
\hline Body Mass Index & & & $1.09(1.01,1.16)$ & $0.023^{*}$ & $1.10(1.02,1.18)$ & $0.014^{*}$ \\
\hline Education (Years) & & & $1.08(0.99-1.16)$ & 0.076 & $1.08(0.99-1.17)$ & 0.064 \\
\hline Diagnosis Duration & & & $1.07(0.90-1.26)$ & 0.443 & $1.07(0.90-1.26)$ & 0.466 \\
\hline Total Comorbidities & & & & & $0.96(0.82-1.11)$ & 0.531 \\
\hline Diabetes Mellitus & & & & & $0.41(0.11-1.59)$ & 0.195 \\
\hline Arthritis & & & & & $0.54(0.14-2.10)$ & 0.373 \\
\hline Total Medications & & & & & $1.06(0.94-1.21)$ & 0.330 \\
\hline Benzodiazepine Use & & & & & $1.49(0.55-4.01)$ & 0.433 \\
\hline Antidepressant Use & & & & & $0.99(0.49-1.98)$ & 0.989 \\
\hline
\end{tabular}


Table 5 Cognitive Scores (ADAS-Cog) at baseline and 18 months by Gait Speed Group at baseline and 18 Months in Mild to Moderate AD. Data presented as means with standard deviations. ADAS-Cog = Alzheimer Disease Assessment Scale - Cognitive Subsection

\begin{tabular}{lllll}
\hline & Baseline Slow Gait Speed & Incident Slow Gait Speed & Normal Gait Speed at Baseline & Normal Gait Speed at 18 Months \\
\hline ADAS-Cog (Baseline) & $37.29+/-9.49$ & $36.47+/-11.56$ & $32.49+/-9.49$ & $36.08+/-11.42$ \\
ADAS-Cog (18 Months) & $44.70+/-16.01$ & $47.28+/-15.91$ & $41.53+/-14.43$ & $40.96+/-14.22$ \\
\hline
\end{tabular}

than threefold increased risk of falls over an 18 month period in those with mild to moderate AD. This is one of the first studies in the literature to assess the longitudinal relationship between slow gait speed and cognitive function in those with a diagnosis of $\mathrm{AD}$

At baseline, the only significant predictors of slow gait speed were greater age and poorer cognition in multivariate analysis. This finding is noteworthy and is largely consistent with previous studies in the literature which have demonstrated a relationship between cognitive function and gait speed in a cross-sectional manner in those with AD [17-19].

The lack of association between baseline slow gait speed and cognitive outcomes is of interest. The only previous study in mild to moderate dementia to assess gait speed and cognition in a longitudinal fashion similarly found no association in longitudinal analysis for general cognition, but found a positive association for particular cognitive sub-domains, namely executive function [21]. One of the reasons for the lack of association in the current study may be that gait was considered as a dichotomous variable based on a pre-specified cut-off. It may be the case that more subtle abnormalities in gait speed may correlate with cognitive function and that such a stringent cut off may mask such an association. Nevertheless, it is possible that whilst slow gait speed may be a predictor of a later diagnosis of dementia, it may lose its predictive value once a diagnosis of dementia has been established.

One of the most interesting findings from the current analysis is the relationship between baseline cognitive function and incident slow gait speed over the duration of the study. Even in a fully adjusted model, baseline cognition was the strongest (apart from age) predictor of incident slow gait speed. This is particularly interesting given that $14 \%$ of the participants with normal gait speed at baseline converted to slow gait speed (incident slow gait speed). Thus, this may represent a subpopulation within those with mild-to-moderate $\mathrm{AD}$ who may be particularly vulnerable to adverse consequences.

The association between baseline slow gait speed and incident falls is particularly stark. Baseline slow gait speed was associated with a greater than threefold increased risk in incident falls over the 18 month study period. Indeed, baseline slow gait speed was the strongest predictor of incident falls under a fully adjusted model. This is particularly pertinent given that more

Table 6 Association between Baseline Slow Gait Speed and Falls at 18 Months in Mild to Moderate AD. Under all models, slow gait speed was significantly associated with falls (dependent variable) risk at 18 months. ADAS-Cog = Alzheimer Disease Assessment Scale - Cognitive Subsection

\begin{tabular}{|c|c|c|c|c|c|c|}
\hline Predictor & Model 1 & & Model 2 & & Model 3 & \\
\hline & IRR (95\% Cl) & $P$ & IRR $(95 \% \mathrm{Cl})$ & $P$ & IRR (95\% Cl) & $P$ \\
\hline Baseline Slow Gait Speed & $3.18(1.97-5.11)$ & $<0.001$ & $3.27(1.96-5.50)$ & $<0.001^{*}$ & $3.09(1.82-5.22)$ & $<0.001^{*}$ \\
\hline Baseline ADAS-Cog & & & $0.95(0.92-0.97)$ & $0.001^{*}$ & $0.95(0.92-0.98)$ & $0.001 *$ \\
\hline Age & & & $1.07(1.04-1.10)$ & $<0.001^{*}$ & $1.07(1.03-1.10)$ & $<0.001^{*}$ \\
\hline Gender (Female) & & & $1.90(1.15-3.11)$ & $0.012^{*}$ & $1.60(0.97-2.65)$ & 0.068 \\
\hline Study Arm & & & $1.20(0.78-1.83)$ & 0.409 & $1.32(0.85-2.06)$ & 0.218 \\
\hline Body Mass Index & & & $0.98(0.94,1.03)$ & 0.489 & $0.96(0.92-1.01)$ & 0.149 \\
\hline Education (Years) & & & $1.07(1.01-1.12)$ & $0.021^{*}$ & $1.10(1.04-1.15)$ & $0.001 *$ \\
\hline Diagnosis Duration & & & $1.02(0.88-1.17)$ & 0.816 & $0.96(0.84-1.11)$ & 0.612 \\
\hline Total Comorbidities & & & & & $0.97(0.88-1.07)$ & 0.527 \\
\hline Diabetes Mellitus & & & & & $1.73(0.85-3.51)$ & 0.128 \\
\hline Arthritis & & & & & $2.19(1.19-4.04)$ & $0.012^{*}$ \\
\hline Total Medications & & & & & $1.03(0.95-1.12)$ & 0.490 \\
\hline Benzodiazepine Use & & & & & $1.05(1.04-3.64)$ & $0.036^{*}$ \\
\hline Antidepressant Use & & & & & $0.67(0.41-1.07)$ & 0.096 \\
\hline
\end{tabular}


than $15 \%$ of the participants with mild to moderate AD experienced at least one fall over the study period. Targeted interventions for falls prevention focussed on those with $\mathrm{AD}$ and slow gait speed are warranted and slow gait speed may be a way to select those with mild to moderate $\mathrm{AD}$ who are particularly vulnerable to experiencing falls, given the adverse consequences of falls in such a cohort [23].

An important consideration in the present study is the nature of the included cohort. Notably, patients with other neurological disorders such as Parkinson's Disease were excluded. Further, patients with a significant cardiac history, previous stroke etc. were all excluded. Thus, the cohort of included patients had fewer comorbidities which may impair cognition than in other naturalistic studies. This enhances the current study in assessing the relationship between gait and cognition specifically in mild-to-moderate AD.

Other strengths of this study include its international nature as well as the high fidelity of follow up in included patients and the large amount of clinical and medical history data available. This enabled us to control for other causes of gait disorders such as arthritis and diabetes in our analysis. Further, it enabled us to include the effects of certain medications on incident slow gait speed and falls, such as benzodiazepines, which have been previously linked with falls in this vulnerable population [32].

Our study has several limitations. Principal amongst these is the fact that gait was assessed as a binary variable. Applying such a stringent cut-off in a dichotomous fashion may not produce the same results as analysis repeated with gait speed as a continuous variable in meters per second for instance. However, in the current study we were limited by the fact that gait speed was recorded as an individual binary variable and not a specific speed in metres per second. This may introduce less accuracy into our results than more detailed recording of gait speeds. Future longitudinal studies should examine the intricacies of the gait-cognition relationship in those with a diagnosis of dementia. A further limitation of the current study is that gait and cognition was only considered over an 18 month period. Such a period may be too short to demonstrate the relationship between gait speed and cognition. Previous studies in the literature have demonstrated a relationship over a longer period of time and we cannot rule out that following this cohort for a longer period of time may demonstrate a significant relationship between baseline gait speed and cognition at follow-up.

In conclusion, we demonstrate a significant baseline association between cognition and slow gait speed in a large cohort of participants with mild to moderate Alzheimer Disease. Poorer baseline cognition was associated with incident slow gait speed over 18 months. However, there was no association between baseline slow gait speed and cognition at follow-up. We also report a significant increase in falls risk over an 18 month period in those with baseline slow gait speed. Further studies should continue to explore the nature of the gaitcognition relationship specifically in those with Alzheimer Disease in helping to tease out which patients may be particularly vulnerable to further cognitive decline and adverse health outcomes.

\section{Abbreviations \\ AD: Alzheimer Disease; ADAS-Cog: Alzheimer's Disease Assessment Scale, Cognitive Subsection; ATC: Anatomic Therapeutic Classification; BMI: Body Mass Index; CDR-Sb: Clinical Dementia Rating, Sum of Boxes; MCl: Mild Cognitive Impairment; NINCDS-ADRDA for AD: National Institute of Neurological and Communicative Disorders and Stroke/Alzheimer's Disease Criteria; NILVAD: A European Multicentre Double Blind-Placebo Controlled Phase III Trial of Nilvadipine in Mild-to-Moderate Alzheimer's Disease}

\section{Acknowledgements}

NILVAD Study Group.

Brian Lawlor, Mercer's Institute for Research on Ageing, St. James's Hospital and Department of Medical Gerontology, Trinity College, Dublin, Ireland; Ricardo Segurado, CSTAR and School of Public Health, Physiotherapy and Sport Science, University College Dublin (UCD), Dublin, Ireland; Sean Kennelly, Department of Age Related Healthcare, Tallaght Hospital, Dublin 24 and Department of Medical Gerontology, Trinity College Dublin; Marcel G. M. Olde Rikkert, Department of Geriatric Medicine, Radboud umc Alzheimer Center, Donders Institute of Medical Neurosciences, Radboudumc, Nijmegen, the Netherlands; Robert Howard, Division of Psychiatry, University College London and King's College London; Florence Pasquier, CHU Lille, Univ. Lille, DISTALZ Laboratory of Excellence, F-59000 Lille, France; Anne Bo"rjesson-Hanson, Department of Psychiatry and Neurochemistry, Institute of Neuroscience and Physiology, Sahlgrenska Academy, University of Gothenburg; Magda Tsolaki, Papanikolaou General Hospital of Thessaloniki, Greece; Ugo Lucca, Laboratory of Geriatric Neuropsychiatry, IRCCS Istituto di Ricerche

Farmacologiche Mario Negri, Milano, Italy; D. William Molloy, University College Cork Centre for Gerontology and Rehabilitation, Cork, Ireland; Robert Coen, Mercer's Institute for Research on Ageing, St. James's Hospital, Dublin, Ireland; Matthias W. Riepe, Department of Geriatrics and Old Age Psychiatry, Psychiatry II, Ulm University at BKH Günzburg, Germany; Ja'nos Ka'Ima'n, Department of Psychiatry, University of Szeged, Hungary; Rose Anne Kenny, Department of Medical Gerontology, Trinity College Dublin (TCD), Dublin, Ireland; Fiona Cregg, Department of Medical Gerontology, Trinity College Dublin (TCD), Dublin, Ireland; Sarah O'Dwyer, Mercer's Institute for Research on Ageing, St. James's Hospital, Dublin, Ireland; Cathal Walsh, Health Research Institute and MACSI, Department of Mathematics and Statistics, University of Limerick, Ireland; Jessica Adams, Department of Old Age Psychiatry, King's College London; Rita Banzi, Laboratory of Geriatric Neuropsychiatry, IRCCS Istituto di Ricerche Farmacologiche Mario Negri, Milano, Italy; Laetitia Breuilh, CHU Lille, Univ. Lille, DISTALZ Laboratory of Excellence, F-59000 Lille, France; Leslie Daly, CSTAR and School of Public Health, Physiotherapy and Sport Science, University College Dublin (UCD), Dublin, Ireland; Suzanne Hendrix, Pentara Corporation, 2180 Claybourne Avenue, Salt Lake City, Utah; Paul Aisen, Department of Neurology, University of Southern California; Siobhan Gaynor, Molecular Medicine Ireland (MMI), Dublin, Ireland; Ali Sheikhi, Health Research Institute and MACSI, Department of Mathematics and Statistics, University of Limerick, Ireland; Diana G. Taekema, Department of Geriatric Medicine, Rijnstate Hospital, Arnhem, the Netherlands; Frans R. Verhey,

Department of Psychiatry and Neuropsychology, School of Mental Health and Neuroscience, Alzheimer Center Limburg, Maastricht University, Maastricht, the Netherlands; Raffaello Nemni, IRCCS Don Gnocchi FoundationUniversity of Milan, Italy; Flavio Nobili, Dept. of Neuroscience (DINOGMI), University of Genoa, and IRCCS AOU Polyclinic, Hospital San Martino, Genoa, Italy; Massimo Franceschi, Neurology Department, Multimedica, Castellanza, Italy; Giovanni Frisoni, Centro San Giovanni di Dio—IRCCS Fatebenefratelli, Brescia, Italy; Orazio Zanetti, Centro San Giovanni di Dio—IRCCS 
Fatebenefratelli, Brescia, Italy; Anastasia Konsta, Aristotle University of ThessaIoniki (AUTH), First Psychiatric Department, Papageorgiou General Hospital, Greece; Orologas Anastasios, Ahepa University General Hospital of Thessaloniki, Greece; Styliani Nenopoulou, Papanikolaou General Hospital of Thessaloniki, Greece; Fani Tsolaki-Tagaraki, Papanikolaou General Hospital of Thessaloniki, Greece; Magdolna Pakaski, Department of Psychiatry, University of Szeged, Hungary; Olivier Dereeper, Centre Hospitalier de Calais, France; Vincent de la Sayette, Centre Hospitalier Universitaire de Caen, France; Olivier Se'ne'chal, Centre Hospitalier de Lens, France; Isabelle Lavenu, Centre Hospitalier de Be'thune, France; Agnès Devendeville, Centre Hospitalier Universitaire d'Amiens, France; Gauthier Calais, Groupement des Ho^pitaux de I'Institut Catholique de Lille (GHICL), France; Fiona Crawford, Archer Pharmaceuticals, Sarasota, Florida, and Roskamp Institute, Sarasota, Florida; Michael Mullan, Archer Pharmaceuticals, Sarasota, Florida, and Roskamp Institute, Sarasota, Florida, Pauline Aalten, PhD, Department of Psychiatry and Neuropsychology, School of Mental Health and Neurosciences, Alzheimer Center Limburg, Maastricht University, Maastricht, the Netherlands; Maria A. Berglund, RN, Sahlgrenska University Hospital, Gothenburg, Sweden; Jurgen A. Claassen MD, PhD, Department of Geriatric Medicine, Radboudumc Alzheimer Center, Donders Institute of Medical Neurosciences, Radboudumc, Nijmegen, the Netherlands; Rianne A. De Heus, MSc, Department of Geriatric Medicine, Radboudumc Alzheimer Center, Donders Institute of Medical Neurosciences, Radboudumc, Nijmegen, the Netherlands; Daan L. K. De Jong, MSc, Department of Geriatric Medicine, Radboudumc Alzheimer Center, Donders Institute of Medical Neurosciences, Radboudumc, Nijmegen, the Netherlands; Olivier Godefroy, MD, PhD, Centre Hospitalier Universitaire d'Amiens, France; Siobhan Hutchinson, MD, St. James's Hospital, Dublin, Ireland; Aikaterini loannou, MD, 1st Department of Neurology, Ahepa University General Hospital, Aristotle University of Thessaloniki, Greece; Michael Jonsson, MD, PhD, Department of Psychiatry and Neurochemistry, Institute of Neuroscience and Physiology, Sahlgrenska Academy, University of Gothenburg, Sweden; Annette Kent, PhD, Trinity College Dublin (TCD), Dublin, Ireland; Jürgen Kern MD, PhD, Department of Psychiatry and Neurochemistry, Institute of Neuroscience and Physiology, Sahlgrenska Academy, University of Gothenburg, Sweden; Petros Nemtsas MD, PhD, 1st Department of Neurology, Ahepa University General Hospital, Aristotle University of ThessaIoniki, Greece; Minoa-Kalliopi Panidou, BSc, MA, 1st Department of Neurology, Ahepa University General Hospital, Aristotle University of Thessaloniki, Greece; Laila Abdullah, PhD, Roskamp Institute, Sarasota, Florida; Daniel Paris, PhD, Roskamp Institute, Sarasota, Florida; Angelina M. Santoso, MD, MSc, Department of Geriatric Medicine, Radboudumc Alzheimer Center, Donders Institute of Medical Neurosciences, Radboudumc, Nijmegen, the Netherlands; Gerrita J. van Spijker, MSc, Department of Geriatric Medicine, Radboudumc Alzheimer Center, Donders Institute of Medical Neurosciences, Radboudumc, Nijmegen, the Netherlands; Martha Spiliotou MD, PhD, 1st Department of Neurology, Ahepa University General Hospital, Aristotle University of ThessaIoniki, Greece; Georgia Thomoglou, BSc, 1st Department of Neurology, Ahepa University General Hospital, Aristotle University of Thessaloniki, Greece; and Anders Wallin, MD, Department of Psychiatry and Neurochemistry, Institute of Neuroscience and Physiology, Sahlgrenska Academy, University of Gothenburg, Sweden.

\section{Authors' contributions}

AHD, BL, SPK: Conception and Design of Secondary Analysis, Statistical Analysis of Results and Writing/Revising the final manuscript. NILVAD Study Group (Principal Investigatory: BL): Design, analysis and conduct of the NILVAD clinical trial. The author(s) read and approved the final manuscript.

\section{Funding}

Funding from the NILVAD study was from the European Commission Framework 7 Programme Health Theme Collaborative Project (grant 279093; PI: Brian Lawlor). The funding agency had no role in the design, recruitment, conduct and data collection of the clinical trial and no input in the analysis, interpretation of data and presentation of primary/secondary trial results.

\section{Availability of data and materials}

Due to the chance of individual participant identification in the current trial, data is not publicly available. Requests to obtain anonymised study data can be addressed to dyera@tcd.ie

\section{Ethics approval and consent to participate}

Ethical approval for the trial was granted from the appropriate National Competent Authorities, Independent Ethics Committees and Institutional Review Boards for all study sites. Ethical Approval for the study also included secondary analysis of NILVAD study data. Written consent was obtained from each participant/caregiver. Individual ethics committees that approved the original trial protocol are as follows: Comite de Protection des Personnes Nord Ouest III, Scientific Council of Papanikolaou Hospital Thessaloniki, Radbound Universitar Medisch Centrum, Concernstaf Kwaliteit en Veiligheid Comissie Mensgebonden Onderzoek Regio Arnhem-Nijmegen, Medical Research Council Ethics Committee for Clinical Pharmacology (KFEB), Comitato Etico Istituzioni Ospedaliere Cattoliche (CEIOC), Comitato Etico IRCCS MultiMedica, Camitato Ethico dell'Aienda Ospedalier a Universitaria S Martino di Genova, Comitato Etico Regione Liguria, Comitato Etico Fondaione Don Carlo Gnocchi.

\section{Consent for publication}

Not applicable.

\section{Competing interests}

The authors declare that they have no competing interests.

\section{Author details}

${ }^{1}$ Department of Age-Related Healthcare, Tallaght University Hospital, Dublin, Ireland. ${ }^{2}$ Department of Medical Gerontology, School of Medicine, Trinity

College Dublin, Dublin 2, Ireland. ${ }^{3}$ Mercer's Institute for Research on Ageing, St James's Hospital, Dublin, Ireland.

\section{Received: 9 July 2019 Accepted: 23 March 2020}

\section{Published online: 30 March 2020}

\section{References}

1. Puvill T, Lindenberg J, Gussekloo J, de AJM C, Slaets JP, RGJ W. Associations of various health-ratings with geriatric giants, mortality and life satisfaction in older people. PLoS ONE. 2016;11. https://doi.org/10.1371/journal.pone. 0163499.

2. Briggs R, O'Neill D. Vascular Gait Dyspraxia. Clin Med (Lond). 2014;14(2):200-2.

3. Liston R, Mickelborough J, Bene J, Tallis R. A new classification of higher level gait disorders in patients with cerebral multi-infarct states. Age Ageing. 2003;32:252-8

4. Verghese J, LeValley A, Hall CB, Katz MJ, Ambrose AF, Lipton RB. Epidemiology of gait disorders in community-residing older adults. J Am Geriatr Soc. 2006;54:255-61.

5. Cesari M, Kritchevsky SB, Penninx BW, Nicklas BJ, Simonsick EM, Newman $A B$, et al. Prognostic value of usual gait speed in well-functioning older people-results from the Health, Aging and Body Composition Study. J Am Geriatr Soc. 2005;53:1675-80 7.

6. Montero-Odasso M, Schapira M, Soriano ER, Varela M, Kaplan R, Camera LA, et al. Gait velocity as a single predictor of adverse events in healthy seniors aged 75 years and older. J Gerontol A Biol Sci Med Sci. 2005;60:1304-9.

7. Verghese J, Wang C, Lipton RB, Holtzer R, Xiaonan X. Quantitative gait dysfunction and risk of cognitive decline and dementia. J Neurol Neurosurg Psychiatry. 2007;78:929-35.

8. Aditya BS, Sharma JC, Allen SC, Vassallo M. Predictors of a nursing home placement from a non-acute geriatric hospital. Clin Rehabil. 2003;17:108-13.

9. Wilson RS, Schneider JA, Beckett LA, Evans DA, Bennett DA. Progression of gait disorder and rigidity and risk of death in older persons. Neurology. 2002;58:1815-9.

10. Hackett RA, Davies-Kershaw H, Orrell CD, Steptoe A. Walking Speed, cognitive Function, and Dementia risk in the English Longitudinal Study of Ageing. 2018;66:1270-5.

11. Dumurgier J, Artaud F, Touraine C, Rouaud O, Tavemier B, Dufouil C, et al. Gait speed and decline in gait speed as predictors of incident dementia. J Gerontol A Biol Sci Med Sci. 2017;72A:655-61.

12. Welmer A-K, Rizzuto D, Qiu C, Caracciolo B, Laukka EJ. Walking speed, processing speed, and dementia: A population-based longitudinal study. J Gerontol A Biol Sci Med Sci. 2014;69A:1503-10 12.

13. Taniguchi $Y$, Kitamura A, Seino S, Murayama H, Amano H, Nofuji Y, et al. Gait performance trajectories and incident disabling dementia among community-dwelling older Japanese. J Am Med Dir Assoc. 2017;18(2):192. e13-20. 
14. Doi T, Tsutsuminoto K, Nakakubo S, Kim MJ, Kurita S, Shimada H. Rethinking the relationship between spatiotemporal gait variables and dementia: a prospective study. J Am Med Dir Assoc. 2019;20(7):899-903.

15. Peel NM, Alapatt LJ, Jones LV, Hubbard RE. The association between gait speed and cognitive status in community-dwelling older people: a systematic review and meta-analysis. J Geront Ser A. 2019;74(6):943-8.

16. Kuate-Tegueu C, Avila-Funes JA, Simo N, LeGoff M, Amieva H, Dartigues JF, et al. Association of Gait Speed, psychomotor speed and dementia. J Alzheimers Dis 2017. 2017;60(2):585-92.

17. Taylor ME, Brodie MA, van Schooten KS, Delbaere K, JCT C, Payne N. (2019) Older People with Dementia Have Reduced Daily-Life Activity and Impaired Daily-Life Gait When Compared to Age-Sex Matched Controls. J Alzheimers Dis. 2019. https://doi.org/10.3233/JAD-181174.

18. Toots ATM, Taylor ME, Lord SR, Close JCT. Associations between gait speed and cognitive domains in older people with cognitive impairment. J Alzheimers Dis. 2019. https://doi.org/10.3233/JAD-18117.

19. Knapstad MK, Steihuag OM, Aaslund MK, Nakling A, Naterstad IF, Fladby T, et al. Reduced walking speed in subjective and mild cognitive impairment: a cross-sectional study. J Geriatr Phys Ther. 2018. https://doi.org/10.1519/JPT. 0000000000000157.

20. Hooghiemstra AM, Ramakers IHGB, Sistermans N, Pignenburg YAL, Aalten P, Hamel REG, et al. Gait speed and grip Srength reflect cognitive impairment and are modestly related to incident cognitive decline in memory clinic patients with subjective cognitive decline and mild cognitive impairment: findings from the 4C study. Jour Gerontol A. 2017;72(6):846-54.

21. Taylor ME, Lasschult DA, Lord SR, Delbaere K, Kurrie SE, Mikolaizak AS, et al. Slow gait speed is associated with executive function decline in older people with mild to moderate dementia: a one year longitudinal study. Arch Gerontol Geriatr. 2017;73:148-53.

22. Allali G, Verghese J. Management of Gait Changes and Fall Risk in $\mathrm{MCl}$ and dementia. Curr Treat Options Neurol. 2017;19:29.

23. Buchner DM, Larson EB. Falls and fractures in patients with Alzheimer-type dementia. JAMA. 1987;257(11):1492.

24. vanDorn C, Gruber-Baldini AL, Zimmerman S, Hebel JR, Port CL, Baumgarten $M$, et al. Dementia is a risk factor for falls and fall injuries in nursing home residents. J Am Geriatr Soc. 2003;51(9):1213-8.

25. Taylor ME, Delbaere K, Lord SR, Mikolaizak AS, Brodaty H, Close JC, et al. Neuropsychological, physical and functional mobility measures associated with falls in cognitively impaired in cognitively impaired older adults. J Gerontol A Biol Sci Med Sci. 2014;69(8):987-95.

26. Taylor ME, Delbaere K, Mikolaizak AS, Lord SR, Close JC, et al. Gait parameter risk factors for falls under simple and dual-task conditions in cognitively impaired older people. Gait Posture. 2013;37(1):126-30.

27. Modarresi S, Divine A, Grahn JA, Overend TJ, Hunter SW, et al. Gait parameters and characteristics associated with increased risk of falls in people with dementia: a systematic review. Int Psychogeriatr. 2018:1-17. https://doi.org/10.1017/S1041610218001783.

28. Lawlor B, Kennelly S, O'Dwyer S, Cregg F, Walsh C, Cohen R, et al. NILVAD protocol: a European multicentre double-blind placebo-controlled trial of nilvadipine in mild-to-moderate Alzheimer's disease. BMJ Open. 2014;4(10): e006364.

29. Meulenbroek O, O'Dwyer S, de Jong D, van Spijker G, Kennelly S, Cregg F, et al. European multicentre double-blind placebo controlled trial of Nilvadipine in mild-to-moderate Alzheimer's disease - the substudy protocols: NILVAD frailty; NILVAD blood and genetic markers; NILVAD cerebrospinal fluid biomarkers; NILVAD cerebral blood flow. BMJ Open. 2016;6(7):e011584

30. Lawlor B, Segurado R, Kennelly S, Olde Rikkert MGM, Howard R, Pasquier F, et al. Nilvadipine in mild to moderate Alzheimer disease: a randomised controlled trial. PLoS Med. 2018;15(9):e21002660.

31. Schott J, Schrag A. What is the clinically relevant change on the ADAS-COG ? J Neurol Neurosurg Psychiatry. 2012;83(2):171-3.

32. Dyer AH, Murphy C, Lawlor B, Kennelly SP, NILVAD Study Group. Cognitive outcomes of long-term benzodiazepine and related drug (BDZR) use in people living with mild to moderate Alzheimer's disease: results from NILVAD. J Am Med Dir Assoc. 2019. https:/doi.org/10.1016/j.jamda.2019.08.006.

\section{Publisher's Note}

Springer Nature remains neutral with regard to jurisdictional claims in published maps and institutional affiliations.

\section{Ready to submit your research? Choose BMC and benefit from}

- fast, convenient online submission

- thorough peer review by experienced researchers in your field

- rapid publication on acceptance

- support for research data, including large and complex data types

- gold Open Access which fosters wider collaboration and increased citations

- maximum visibility for your research: over $100 \mathrm{M}$ website views per year

At $\mathrm{BMC}$, research is always in progress.

Learn more biomedcentral.com/submissions 